\title{
Cómo la Web of Science (WoS) de Thomson Reuters habría confundido dos revistas distintas con el mismo título durante años: el caso de "Journal of Public Relations Research"
}

\author{
Kathy Matilla*, Marc Compte-Pujol**, Salvador Hernández*** \\ *Universidad Ramon Llull, Barcelona. España \\ **Universidad de Vic - Universidad Central de Cataluña. Vic. Barcelona. España \\ ***Universidad Católica San Antonio de Murcia, Murcia. España \\ Correos-e: kathyms@blanquerna.url.edu,marc.compte@uvic.cat,shernandez@ucam.edu
}

Recibido: 08-09-2015; 2a versión: 31-12-2015; Aceptado: 21-01-2016.

Cómo citar este artículo/Citation: Matilla, K.; Compte-Pujol, M.; Hernández, S. (2016). Cómo la Web of Science (WoS) de Thomson Reuters habría confundido dos revistas distintas con el mismo título durante años: el caso de "Journal of Public Relations Research". Revista Española de Documentación Científica, 39(2): e132. doi: http://dx.doi.org/10.3989/redc.2016.2.1332

Resumen: Descripción del proceso mediante el cual se habría detectado una notable anomalía en la base de datos bibliográficos de la Web of Science (WoS). Concretamente, desde el año 2002 la WoS habría confundido y entremezclado aleatoriamente numerosos artículos de dos revistas distintas, ambas especializadas en relaciones públicas, una norteamericana y otra surcoreana, que comparten un mismo título: Journal of Public Relations Research. El caso es especialmente relevante al tratarse de dos publicaciones escritas en idiomas totalmente distintos (inglés y coreano), con diferentes números de ISSN y con distintas líneas editoriales, en cuyos respectivos volúmenes no coinciden ni autores, ni contenido.

Palabras clave: Bibliometría; Corea del Sur; Estados Unidos de América; Journal Citations Reports (JCR); Journal of Public Relations Research; relaciones públicas; Social Sciences Citation Index (SSCI); Thomson Reuters; Web of Science (WoS).

How the Web of Science confused two different journals with the same title for many years: the case of the "Journal of Public Relations Research"

Abstract: A description of how a conspicuous anomaly in the Web of Science (WoS) database was detected. Beginning in 2002 the WoS randomly mistook and interspersed numerous articles from two different journals, one American and the other South Korean, both specialized in public relations and sharing the same title: Journal of Public Relations Research. The case is especially noteworthy since these are two publications written in very different languages (English and Korean), with different ISSN numbers and editorial outlooks, and whose respective volumes share neither authors nor content.

Keywords: Bibliometrics; South Korea; Journal Citations Reports (JCR); Journal of Public Relations Research; public relations; Social Sciences Citation Index (SSCI); Thomson Reuters; United States of America; Web of Science (WoS).

Copyright: () 2016 CSIC. Este es un artículo de acceso abierto distribuido bajo los términos de la licencia Creative Commons Attribution-Non Commercial (by-nc) Spain 3.0. 


\section{INTRODUCCIÓN}

El número de artículos académicos especializados en bibliometría de las Relaciones Públicas (en adelante RP) se ha ido visto progresivamente incrementado conforme ha ido pasando el tiempo. Se observa dicho aumento durante la última década previa al siglo XXI, que continua en el presente (Ferguson, 1984; Pasadeos y Renfro, 1989, 1992; McElreath y Blamphin, 1994; Morton y Lin, 1995; Pasadeos y otros, 1999; Duffy, 2000; Sallot y otros, 2003; Botan y Taylor, 2004; Ki y Shin, 2006; Xifra y Castillo, 2006; Hoy y otros, 2007; Cheng y De Gregorio, 2008; Pasadeos y otros, 2010; Castillo y Carretón, 2010; Pasadeos y otros, 2011; Seon-Kyoung y I-Huei, 2012; Ye y $\mathrm{Ki}, 2012$; Fussell y otros, 2013; Davidson, 2014; Kim y otros, 2014; Míguez y otros, 2014; y Marca y otros, 2015, entre otros).

Dichos estudios bibliométricos han permitido compartir periódicamente las tendencias en investigación en RP, tanto a nivel de técnicas, como de temáticas y de corrientes de pensamiento. Asimismo, han servido para demostrar que, en las últimas décadas, ha aumentado no sólo la cantidad de artículos publicados sobre RP, sino también su relevancia científica y el número de autores internacionales especializados en este campo del conocimiento.

En este sentido, cabe señalar que de las 78 revistas catalogadas en la base de datos Social Sciences Citation Index-Communication-Journal List de Thomson Reuters, únicamente 2 están dedicadas a las RP como dominio particular de la comunicación: Public Relations Review (también indizada en Scopus) y Journal of Public Relations Research (búsquedas realizadas con fecha 20.07.15 en ambos casos). Ambas revistas están, asimismo, indizadas en Journal Citation Reports $(J C R)$, por lo que es muy frecuente que los estudios bibliométricos especializados en RP utilicen únicamente estas dos revistas como muestra (Seon-Kyoung y I-Huei, 2012).

\subsection{Objetivos de investigación}

El objetivo principal de este estudio es señalar y difundir un importante error detectado en la WoS de Thomson Reuters mientras se llevaba a cabo una investigación bibliométrica focalizada en revistas especializadas en RP con alto factor de impacto.

Para ello, se detallará el proceso mediante el cual se ha podido observar una anomalía que afecta desde hace más de una década a los resultados de búsqueda de la revista Journal of Public Relations Research que, junto a la revista Public Rela- tions Review, es una de las dos principales revistas académicas internacionales especializadas en RP y, debido a su indización $J C R$, de las más citadas por la comunidad científica.

\section{METODOLOGÍA Y LIMITACIONES}

Inicialmente, nuestro estudio pretendía investigar, de forma sistemática, los artículos sobre estrategia aplicada a las RP publicados en las revista académicas norteamericanas Journal of Public Relations Research y Public Relations Review durante el periodo acotado entre el 1 de enero de 2000 y el 20 de julio de 2015, analizando diversas variables desde una perspectiva bibliométrica. Para ello se definieron los parámetros de búsqueda "strategy" y "strategic" en el título del artículo, los resúmenes y las palabras clave, ya que nos interesaba analizar exclusivamente los artículos, como unidad de análisis, que planteasen una perspectiva estratégica en sus contenidos.

Sin embargo, se comprobó que Journal of Public Relations Research no ofrecía información sobre las palabras clave en su web corporativa, lo cual constituyó la primera limitación de la investigación, que nos condujo a su vez a trasladar la búsqueda a la base de datos multidisciplinar de la WoS para examinar si, desde allí, era posible disponer de dicha información.

Resultó sorprendente comprobar que, desde la WoS, de tanto en tanto aparecían numerosas entradas de artículos escritos en caracteres orientales que, posteriormente comprobamos que eran coreanos, hecho que se nos antojó extraño en una revista norteamericana que, por otra parte, conocíamos bien por haber citado en otros lugares buena parte de sus artículos, consultados siempre desde su web corporativa y sin haber identificado artículo alguno publicado en dicha lengua asiática.

En primera instancia se consideró que pudiera tratarse de una edición traducida al coreano de la revista norteamericana, con distribución en Corea del Sur. Para dilucidar la duda se acudió al buscador Google, identificando las entradas a sus respectivas webs corporativas y comprobando que se trataba de dos publicaciones diferentes, una editada por Routledge en Estados Unidos y la otra en la República de Corea del Sur por la Korean Academic Society for Public Relations pero que, en ambos casos, ostentaban el mismo título -en inglés-: Journal of Public Relations Research.

Detectada y verificada la anomalía, se procedió a analizar hasta qué punto y durante cuanto tiempo la WoS habría podido confundir ambas revistas en su base de datos para académicos. 


\section{RESULTADOS}

El 20 de julio de 2015 se comprueba que, al realizar una búsqueda básica en la base de datos de la WoS -a través de las opciones "Nombre de publicación" y período de tiempo "Todos los años"-, aparece listada una única revista con el título de Journal of Public Relations Research, la cual genera 388 resultados de artículos publicados.

Los artículos de dicho listado se presentan numerados y ordenados cronológicamente, en sentido descendente, de los más recientes a los más antiguos. Sin embargo, dichos resultados no corresponden únicamente a los artículos de la revista norteamericana, sino que aparecen mezclados con los de la revista surcoreana, sin especificar que se trata de otra revista diferente que posee idéntico título. Tras una lectura detenida resultó sencillo diferenciar la procedencia de unos artículos y de otros porque se pudo apreciar, de un lado, que los volúmenes de la norteamericana van por delante de la coreana (ej. : en 2012 la editada por Routledge iba por el vol. 23, mientras que la surcoreana alcanzaba el vol. 16) y, de otro, porque la oriental presenta los nombres de los autores y el nombre de la revista (홍보학연구) en caracteres coreanos, además de en inglés.

Por ello, procedimos a comparar las características editoriales de ambas revistas a partir de la información disponible en sus webs corporativas [Ver Tabla I], demostrando que comparten únicamente el título.

Se comprobó que ambas revistas se crearon con 8 años de diferencia: en 1989 la americana y en 1997 la surcoreana. Cuando nació la surcoreana, la norteamericana ya había cambiado su denominación inicial de Public Relations Research Annual a Journal of Public Relations Research (1992).

Ambas revistas están promovidas por asociaciones académicas: la norteamericana Public Relations Division de la AEJMC - Association for Education in Journalism and Mass Communication y la coreana KASPR - Korean Academic Society for Public Relations, respectivamente.

Se destaca que la editada por Routledge recibe el apoyo de las principales asociaciones profesionales estadounidenses: ICA - International Communication Association; NCA - National Communication Association; PRSA - Public Relations Society of America; e IABC -International Association of Business Communicators. La revista norteamericana aparece indizada en la categoría de comunicación del SSCI y ocupa la posición 50 de 76 en $J C R$, con un factor de impacto de 0,556. También está indizada en Scopus/SCImago. Por su parte, la revista surcoreana está indizada en el KCI-Korean Citation Index.
Posteriormente, tras depurar los 388 resultados de búsqueda obtenidos mediante la base de datos WoS -eliminando 5 artículos duplicados de la versión coreana en 2002 y excluyendo call for papers, erratums y editorial boards-, obtuvimos un total de 378 artículos publicados hasta el 20 de julio de 2015 .

El primer artículo recogido en la base de datos de la WoS para Journal of Public Relations Research data del año 2002 y corresponde al vol. 6, número 1 , de la revista surcoreana. En esta línea, cabe señalar que los 119 artículos más antiguos de Journal of Public Relations Research publicados en WoS corresponden exclusivamente a la revista surcoreana -creada en 1997- y que no es hasta el año 2008 que se identifica un primer artículo perteneciente a la revista norteamericana, que lleva publicándose ininterrumpidamente desde 1989 [Ver Tabla II].

Se observa que en 2015 no aparece ninguna entrada de artículos de la revista surcoreana, mientras que en 2014 y en 2013 sí aparecen consignadas entradas coreanas, pero únicamente de 3 artículos cada año. Sin embargo, en la web corporativa de la revista surcoreana los volúmenes editados esos mismos años sí que aparecen completos.

Se comprueba que el vol. 16 (2012) de la revista surcoreana es el último que aparece completo en la WoS (numeración del 98 al 121). Se constata que a partir del vol. 17, número 1 (2013) de la revista surcoreana no se listan todos sus artículos en el SSCI de la WoS, sino únicamente un porcentaje ínfimo. De hecho, en dicho vol. 17(1) hay una única entrada (núm. 66) y en el vol. 17(2) solamente aparecen 2 (numeración del 67 y 68).

Se desconoce el criterio seguido por la WoS para la selección de tan escaso e incompleto número de artículos de la revista surcoreana en los años 2014 y 2013. Adicionalmente, el hecho de que en los primeros seis meses y medio de 2015 no se haya listado ningún artículo coreano, unido a que no aparece ninguna referencia a Thomson Reuters en la web corporativa de la revista surcoreana en la búsqueda de 2015, quizás indique que en algún momento ha sido excluida de la base de datos de la WoS, aunque no es posible afirmarlo con seguridad al no haber recibido respuesta escrita a la consulta realizada a la revista solicitando dicha información, que formulamos asimismo por escrito, en inglés, mediante correo electrónico.

Finalmente, apuntamos como curiosidad el hecho de que la WoS haya suprimido en su base de datos un montante coincidente y exacto de 89 artículos por cada revista, aunque durante períodos diametralmente opuestos. 
Tabla I. Comparativa de las características editoriales de la revista Journal of Public Relations Research (USA) y de la revista Journal of Public Relations Research (Corea del Sur)

\begin{tabular}{|c|c|c|}
\hline & Journal of Public Relations Research (USA) & $\begin{array}{c}\text { Journal of Public Relations Research } \\
\text { (Corea del Sur) }\end{array}$ \\
\hline $\begin{array}{l}\text { Año de } \\
\text { creación }\end{array}$ & $\begin{array}{c}1989 \\
\text { con el nombre de Public Relations Research Annual. A } \\
\text { partir de } 1992 \text { pasó a denominarse Journal of Public } \\
\text { Relations Research }\end{array}$ & $\begin{array}{l}1997 \\
\text { con el nombre de 홍보학 연구 (Journal of } \\
\text { Public Relations Research). No ha sufrido } \\
\text { ningún cambio de titulación desde entonces. }\end{array}$ \\
\hline $\begin{array}{l}\text { Link de acceso } \\
\text { a la web cor- } \\
\text { porativa }\end{array}$ & $\frac{\text { http://www.tandfonline.com/action/journalInformation?s- }}{\underline{\text { how }=\text { aimsScope\&journalCode }=\text { hprr20 }}}$ & $\frac{\text { http://kiss.kstudy.com/journal/list name. }}{\text { asp?key } 1=25404 \& \text { key } 2=3086}$ \\
\hline País & Estados Unidos de América & Corea del Sur \\
\hline Editorial & Routledge (Taylor \& Francis) & $\begin{array}{l}\text { Korean Academic Society for Public } \\
\text { Relations (http://www.kaspr.net) }\end{array}$ \\
\hline $\begin{array}{l}\text { Frecuencia de } \\
\text { publicación }\end{array}$ & 5 volúmenes/año & 4 volúmenes/año \\
\hline Editor & Karen Russell (Universidad de Georgia) & No se indica \\
\hline ISSN & $\begin{array}{l}\text { 1062-726X (Impreso) } \\
\text { 1532-754X (Online) }\end{array}$ & 1229-2869 (Online) \\
\hline $\begin{array}{l}\text { Asociación } \\
\text { promotora }\end{array}$ & $\begin{array}{l}\text { Public Relations Division de la AEJMC-Association for } \\
\text { Education in Journalism and Mass Communication }\end{array}$ & $\begin{array}{l}\text { KASPR-Korean Academic Society for } \\
\text { Public Relations }\end{array}$ \\
\hline $\begin{array}{l}\text { En colabora- } \\
\text { ción con }\end{array}$ & $\begin{array}{c}\text { ICA-International Communication Association; NCA- } \\
\text { National Communication Association; PRSA-Public } \\
\text { Relations Society of America; IABC-International } \\
\text { Association of Business Communicators }\end{array}$ & $\begin{array}{l}\text { No se indica la existencia de ninguna } \\
\text { asociación colaboradora }\end{array}$ \\
\hline Objetivos & $\begin{array}{l}\text { The JPRR publishes scholarship that creates, tests, } \\
\text { or expands public relations theory. Manuscripts may } \\
\text { examine why organizations practice public relations } \\
\text { as they do and how public relations can be conducted } \\
\text { more effectively; analysis of the publics of public } \\
\text { relations; scholarly criticism of public relations } \\
\text { practice; and development of the history, ethics, or } \\
\text { philosophy of public relations. Because of the wide } \\
\text { range of influences on and effects of public relations, } \\
\text { interdisciplinary research is particularly encouraged. } \\
\text { Two kinds of articles can be submitted: reviews of } \\
\text { major programs of research (20-60 double-spaced } \\
\text { manuscript pages) and reports of original research } \\
\text { (approximately } 20 \text { double-spaced manuscript pages). } \\
\text { All methodologies are appropriate, including critical, } \\
\text { historical, legal, philosophical, and social scientific.- }\end{array}$ & No se indican objetivos \\
\hline Temas & $\begin{array}{c}\text { Business, Management and Accounting; } \\
\text { Communication Studies; Economics, Finance, } \\
\text { Business \& Industry; Humanities; Marketing; } \\
\text { Marketing Communications; Media \& } \\
\text { Communications; Media \& Film Studies; Public } \\
\text { Relations; Public Relations in Media. }\end{array}$ & Mass Communication \\
\hline Indización & $\begin{array}{l}\text { ComIndex; ComAbstracts; Communication Abstracts; } \\
\text { Communication Institute for Online Scholarship; Index } \\
\text { to Journals in Mass Communication; LEXIS/NEXIS; } \\
\text { PsycINFO; EBSCO Host Products y Social Sciences } \\
\text { Citation Index (2014 Journal Citations Report - } 50 \text { de } 76 \\
\text { categoría Comunicación (Ciencias Sociales) - FI 0,556). }\end{array}$ & $\begin{array}{l}\text { KCI-Korean Journal Database (Korean } \\
\text { Citation Index); Korean Studies } \\
\text { Information Service System (KISS); } \\
\text { Books I\&I; Booktory; CardQ; Directory of } \\
\text { Korea Open Access Journals; StopBook. }\end{array}$ \\
\hline
\end{tabular}


Tabla II. Total de artículos publicados de las revistas Journal of Public Relations Research (USA) y Journal of Public Relations Research (Corea del Sur) por año de publicación y soporte

\begin{tabular}{|c|c|c|c|c|c|c|}
\hline \multirow[b]{2}{*}{$\begin{array}{c}\text { Año de } \\
\text { publicación }\end{array}$} & \multicolumn{3}{|c|}{ Revista americana (Routledge) } & \multicolumn{3}{|c|}{ Revista sudcoreana (KASPR) } \\
\hline & En WoS & $\begin{array}{c}\text { En web } \\
\text { corporativa }\end{array}$ & Diferencia & En WoS & $\begin{array}{c}\text { En web } \\
\text { corporativa }\end{array}$ & Diferencia \\
\hline 2002 & 0 & 12 & -12 & 15 & 15 & $\mathbf{0}$ \\
\hline 2003 & 0 & 11 & -11 & 19 & 19 & $\mathbf{0}$ \\
\hline 2004 & 0 & 11 & -11 & 20 & 20 & $\mathbf{0}$ \\
\hline 2005 & 0 & 20 & -20 & 13 & 13 & $\mathbf{0}$ \\
\hline 2006 & 0 & 19 & -19 & 16 & 16 & $\mathbf{0}$ \\
\hline 2007 & 0 & 16 & -16 & 20 & 20 & $\mathbf{0}$ \\
\hline 2008 & 19 & 19 & 0 & 11 & 11 & $\mathbf{0}$ \\
\hline 2009 & 25 & 25 & 0 & 11 & 11 & $\mathbf{0}$ \\
\hline 2010 & 27 & 27 & $\mathbf{0}$ & 29 & 28 & 1 \\
\hline 2011 & 15 & 15 & $\mathbf{0}$ & 20 & 20 & $\mathbf{0}$ \\
\hline 2012 & 23 & 23 & $\mathbf{0}$ & 24 & 24 & $\mathbf{0}$ \\
\hline 2013 & 22 & 22 & $\mathbf{0}$ & 3 & 33 & -30 \\
\hline 2014 & 25 & 25 & 0 & 3 & 44 & -41 \\
\hline $2015^{*}$ & 18 & 18 & 0 & 0 & 19 & -19 \\
\hline Total & 174 & 263 & -89 & 204 & 293 & -89 \\
\hline
\end{tabular}

* Hasta el 20 de julio de 2015.

Por otra parte, se puede resaltar también el hecho de que en los datos del año 2010 de la revista coreana se contabiliza en WoS un artículo más que en la propia web de la revista (29 artículos frente a 28).

\section{DISCUSIÓN Y CONCLUSIONES}

En primer lugar, se destaca como observación relevante que ninguno de los estudios realizados anteriormente sobre bibliometría de las RP haya hecho referencia alguna a la peculiar irregularidad que la WoS parece haber cometido durante más de una década en las entradas referentes a Journal of Public Relations Research, una de las dos únicas revistas especializadas en la disciplina de las RP que se encuentran indizadas en el Social Sciences Citation Index de Thomson Reuters, junto con la norteamericana Public Relations Review.

Este hecho permite especular en torno a la posibilidad de que sus autores hayan acudido directamente a las webs corporativas de las revistas para llevar a cabo sus respectivos trabajos de campo, en lugar de acceder en sus búsquedas desde la web del WoS, motivo por el cual la anomalía ahora identificada no habría sido observada con anterioridad.
A partir del descubrimiento de la anomalía descrita, futuras investigaciones se hacen necesarias y podrían centrarse en analizar aquellos trabajos bibliométricos -únicamente los realizados desde 2002- que han incluido en su muestra a las ediciones americana o surcoreana de Journal of Public Relations Research, para comprobar si el posible error de WoS hubiera podido influir negativamente en el proceso metodológico de alguno de ellos, provocando alteraciones en los resultados de los respectivos trabajos de campo $\mathrm{y}$, por consiguiente, también en las discusiones $\mathrm{y}$ conclusiones.

A modo de conclusión, confiamos que este estudio llegue al conocimiento de los gestores de la WoS para que se solvente esta posible anomalía -que nos atrevemos a catalogar de inadmisibleen la que se considera como la base de datos de referencia para el colectivo internacional de investigadores y académicos y que, paralelamente, sea tenido en cuenta en futuros estudios bibliométricos sobre RP que incluyan a una o a ambas revistas en sus objetos de estudio. 


\section{REFERENCIAS}

Botan, C.H.; Taylor, M. (2004). Public Relations: State of the Field. Journal of Communication, vol. 54 (4), 645-661. http://dx.doi. org/10.1111/j.1460-2466.2004.tb02649.x

Castillo, A.; Carretón, Ma C. (2010). Investigación en Comunicación. Estudio bibliométrico de las revistas de Comunicación en España. Comunicación y Sociedad, vol. 13 (2), 289-327.

Cheng, I-H.; de Gregorio, F. (2008). Does (linking with) practice make perfect?: A survey of public relations scholars' perspectives. Journal of Public Relations Research, vol. 20, 377-402. http:// dx.doi.org/10.1080/10627260802153298

Davidson, S. (2014). Everywhere and nowhere: Theorising and researching public affairs and lobbying within public relations scolarship. Public Relations Review, vol. 41 (5), 615-627. http://dx.doi. org/10.1016/j.pubrev.2014.02.023

Duffy, M. (2000). There's no two-way symmetric about it: A postmodern examination of public relations textbooks. Critical Studies in Media Communication, vol. 17 (3), 294-315. http:// dx.doi.org/10.1080/15295030009388397

Ferguson, M.A. (1984). Building Theory in Public Relations: Interorganizational Relationships. Annual Convention of the Association of Journalism and Mass Communication, Agosto 1984, Gainesville, FL.

Fussell Sisco, H.; Pressgrove, G.; Collins, E.L. (2013). Paralleling the Practice: An Analysis of the Scholarly Literature in Nonprofit Public Relations, Journal of Public Relations Research, vol. 25 (4), 282-306. http://dx.doi.org/10.1080/1062726X.2013.806869

Hoy, P.; Raaz, O; Wehmeier, S. (2007). From facts to stories or from stories to facts? Analyzing public relations history in public relations textbooks. Public Relations Review, vol. 33, 191-200. http:// dx.doi.org/10.1016/j.pubrev.2006.11.011

Journal of Public Relations Research (EEUU). Web corporativa. Disponible en: http://www.tandfonline. com/action/journalInformation?show=aimsScope\& journalCode=hprr20 [consultado: 2015-07-20].

Journal of Public Relations Research (Corea delSur). Web corporativa. Disponible en: http://kiss.kstudy.com/ journal/list_name.asp?key $1=25404 \&$ key $2=3086$ [consultado: 2015-07-20].

Ki, E.-J.; Shin; J.-H. (2006). Status of organizationpublic relationship research from an analysis of published articles, 1985-2004. Public Relations Review, vol. 32 (2), 194-195. http://dx.doi. org/10.1016/j.pubrev.2006.02.019

Kim S.-Y.; Choi, M.-I.; Reber, B.H.; Kim, D. (2014). Tracking public relations scholarship trends: Using semantic network analysis on PR Journals from 1975 to 2011. Public Relations Review, vol. 40 (1), 116-118. http://dx.doi.org/10.1016/j.pubrev.2013.11.017
Korean Academic Society for Public Relations. Web corporativa. Disponible en: http://www.kaspr.net [consultado: 2015-07-26].

Marca-Francés, G.; Matilla, K.; Ortiga-Fontgivell, B. (2015). Research on health communication and public relations with an ISI bibliometric analysis from 2001 to 2013: A comparative study. Mitteilungen Klosterneuburg Journal, vol. 65 (1), section 1, 34-44.

McElreath, M.; Blamphin, J. (1994). Partial answers to priority research questions -and gaps-found in the Public Relations Society of America's Body of Knowledge, Journal of Public Relations Research 6 (2), 69-104. http://dx.doi.org/10.1207/s1532754xjprr0602_01

Míguez-González, M.I.; Baamonde-Silva, X.M.; Corbacho-Valencia, J.M. (2014). A bibliographic study of public relations in Spanish media and communication journals, 2000-2012, Public Relations Review, vol. 40 (5), 818-828. http://dx.doi. org/10.1016/j.pubrev.2014.08.002

Morton, L. ; Lin, L. (1995). Content and citation analyses of Public Relations Review. Public Relations Review, 21(4), 337-350. http://dx.doi.org/10.1016/03638111(95)90117-5

Pasadeos, Y.; Renfro, B. (1989). A citation study of public relations research 1975-1986. Public Relations Review, vol. 15 (3), 48-50. http://dx.doi.org/10.1016/S03638111(89)80004-9

Pasadeos, Y.; Renfro, B. (1992). A bibliometric analysis of public relations research. Journal of Public Relations Research, vol. 4 (3), 167-183. http:// dx.doi.org/10.1207/s1532754xjprr0403_03

Pasadeos, Y.; Renfro, B.; Hanily, M. (1999). Influential authors and works of public relations scholarly literature: A network of recent research. Journal of Public Relations Research, vol. 11 (1), 29-52. http:// dx.doi.org/10.1207/s1532754xjprr1101_02

Pasadeos, Y.; Berger, B.; Renfro, B. (2010). Public relations as a maturing discipline: An update on research networks. Journal of Public Relations Research, vol 22 (2), 136-158. http://dx.doi. org/10.1080/10627261003601390

Pasadeos, Y.; Lamme, M.O.; Gower, K.; Tian, S. (2011). A methodological evaluation of public relations research. Fuel and Energy Abstracts, vol. 37 (2), 163-165. http://dx.doi.org/10.1016/j. pubrev.2011.01.007

Sallot, L.M.; Lyon, L.J.; Acosta-Alzuru, C.; Jones, K.O. (2003). From Aardvark to zebra: A new millennium analysis of theory development in public relations academic journals. Journal of Public Relations Research, vol. 15 (1), 27-90. http://dx.doi. org/10.1207/S1532754XJPRR1501_2

Seon-Kyoung, A.; I-Huei Ch. (2012). Crisis Communication Research in Public Relations Journals. Tracking Research Trends Over Thirty 
Years. En T. Coombs y S.J. Holladay (Eds.), The Handbook of Crisis Communication (65-90). Chichester, UK: Wiley-Blackwell.

Social Sciences Citation Index - Communication Journal List. Web corporativa. Disponible en: http:// science.thomsonreuters.com/cgi-bin/jrnlst/jlresults. cgi?PC=SS\&SC=EU [consultado: 2015-07-20].

Xifra, J.; Castillo, A. (2006). Forty Years of Doctoral Public Relations Research in Spain: A Quantitative
Study of Dissertation Contribution to Theory Development, Public Relations Review, vol. 32, 302-308.

Ye, L.; Ki, E.-J. (2012). The Status of Online Public Relations Research: An Analysis of Published Articles in 1992-2009, Journal of Public Relations Research, vol. 24 (5), 409-434. http://dx.doi.org/10.1080/10 62726X.2012.723277 